\title{
DEL VIVIR BIEN Y DEL DESARROLLO SUSTENTABLE. EXTRACTIVISMOS Y CONSTRUCCIÓN DE ALTERNATIVAS AL DESARROLLO EN BOLIVIA Y CHILE
}

\author{
ON LIVING WELL AND SUSTAINABLE DEVELOPMENT. \\ EXTRACTIVISM AND THE CONSTRUCTION OF ALTERNATIVES \\ TO DEVELOPMENT IN BOLIVIA AND CHILE
}

\author{
Miriam Lehnert ${ }^{*}$ y Noelia Carrasco**
}

\begin{abstract}
Considerando las trayectorias de los modelos de Vivir Bien en Bolivia y del desarrollo sustentable en Chile, el artículo examina las características sociopolíticas y socioculturales de los procesos de desarrollo en América Latina en la actualidad. En ambos casos, los enfoques para definir al desarrollo son considerados como el resultado de procesos políticos y económicos nacionales y globales, donde las premisas tradicionales del desarrollo exclusivamente determinado por el crecimiento económico, han sido ajustadas. Ambas propuestas plantean la eventual renovación de las orientaciones y condiciones materiales del desarrollo. No obstante, ambas propuestas siguen estando condicionadas por el extractivismo, suscitando nuevas formas de conflictos y resistencias por parte de comunidades locales y movimientos sociales. La pregunta central que se aborda en el presente texto es ¿de qué manera estos modelos que inicialmente se propusieron responder a las críticas al desarrollo sin identidad ni resguardos sociales y ambientales, se enfrentan hoy a su propia crisis? ¿Cuáles son las resistencias al Vivir Bien y al desarrollo sustentable en América Latina, que responden críticamente a la profundización de las actividades extractivistas en los países latinoamericanos durante la última década?

Luego de una contextualización general de ambos casos, se exponen los principales contenidos de la propuesta del Vivir Bien en Bolivia y los usos políticos del desarrollo sustentable en Chile. Posteriormente se hace referencia a las dinámicas territoriales construidas en cada país, desde ángulos locales críticos que apelan a su rectificación y a transformaciones profundas de las bases económico-políticas que regulan al desarrollo. Si bien este no constituye objetivamente un trabajo de tipo comparativo, permitirá conocer importantes diferencias y también coincidencias en las configuraciones económicas y culturales del desarrollo en dos países latinoamericanos.
\end{abstract}

Palabras claves: Crisis del desarrollo, territorios, resistencias, naturalezas, desarrollos otros.

Considering the trajectories of the models of Vivir Bien in Bolivia and sustainable development in Chile, this article examines the socio-political and socio-cultural characteristics of the development processes in Latin America today. In both cases, the approaches defining development are considered the result of national and global political and economic processes, where the traditional premises of development exclusively determined by economic growth have been adjusted. Both proposals suggest the eventual renewal of the orientations and material conditions of development. However, both proposals are still conditioned by extractivism, raising new forms of conflicts and resistances by local communities and social movements nowadays. This text central question is: how do these models, which initially set out to respond to criticisms of development without identity, neither social nor environmental safeguards, face their own crisis nowadays? What are the forms of resistance against living well and sustainable development in Latin America that respond critically to the deepening of extractive activities in Latin American countries during the last decade? After a general contextualization of both cases, the main contents of the proposal of Living Well in Bolivia and the political uses of sustainable development in Chile are presented. Subsequently, reference is made to the territorial dynamics built in each country, from critical local angels that appeal to its rectification and to deeper transformations of the political, economic bases that regulate development. Although this does not strictly constitute a comparative work, it will allow for knowing important differences, and coincidences in the economic and cultural configurations of development in two Latin American countries.

Key words: Development crisis, territories, resistances, natures, other developments.

\footnotetext{
* Universidad Goethe Frankfurt am Main, Estudiante de Doctorado, Departamento de Sociología. Frankfurt am Main, Alemania. Correo electrónico: m.lehnert@em.uni-frankfurt.de

** Universidad de Concepción, Departamento de Historia. Chile. Correo electrónico: noeliacarrasco@udec.cl
}

Recibido: 20 de noviembre de 2019. Aceptado: 22 de septiembre de 2020. 


\section{Introducción}

Chile y Bolivia son dos países de América del Sur con históricos conflictos limítrofes, especialmente marcados por el objetivo de Bolivia de recuperar acceso soberano al mar. La historia de cada país ha estado marcada por procesos políticos y culturales independientes, y en las últimas décadas se expresaron evidentes diferencias entre las políticas del gobierno liderado por Evo Morales en Bolivia y los gobiernos socialdemócratas y de derecha que han estado en el poder en Chile. En el ámbito del desarrollo económico y social, cada país ha implementado políticas y programas basados en proyectos económicos con importantes diferencias, especialmente en lo que respecta a la participación del Estado en el control de recursos. No obstante, las políticas económicas de ambos países coinciden en la profundización del modelo extractivo a gran escala (Bebbington, 2009; Villalba-Eguiluz y Etxano, 2017), suscitando una secuencia intensiva de conflictos territoriales y la detonación de crisis políticas y sociales que en Bolivia incluyen un reciente golpe de Estado y en Chile el inicio de un proceso deliberativo para modificar la Constitución. La selección de ambos países como casos de estudio, se basa en que se consideran representativos de la multiplicidad de cauces que pueden adoptar los modelos y procesos de desarrollo en América Latina en la actualidad. Es decir, una región donde países con tradiciones políticas muy diferentes y con fundamentos abiertamente disímiles en sus cartas constitucionales vigentes, se enfrentan de igual manera a crisis suscitadas por la resistencia a las transformaciones por parte de sectores conservadores, que detentan poderes e intereses económicos y culturales de raigambre moderna y global. Así también, en ambos casos se expresa una dimensión muy presente en América Latina hoy, que identificamos aquí como las bases históricas y socioculturales de esta crisis, revelada por la población que confronta al modelo extractivo y reivindica otras formas de desarrollo (Escobar, 2014; Navia López, 2019; Rivera Cusicanqui, 2018). Un ejemplo que ha adquirido atención mundial en este sentido, y que ilustra la importancia de seleccionar ambos casos, ha sido el del litio que, siendo un mineral clave para las energías renovables y la fabricación de baterías para autos eléctricos, es un recurso natural sujeto a la intensa extracción tanto en Chile como en Bolivia (Fundación Solón, 2019; Lombardi, 2018). En ambos casos esta actividad ha suscitado la reacción de comunidades locales y movimientos socioambientales que, con independencia de si el desarrollo potencie una industria nacional o transnacional, rechazan toda actividad que suponga la intervención intensiva de los recursos naturales.

Los objetivos de este trabajo son: a. Mostrar cómo ambos países, habiendo utilizado fórmulas distintas para definir sus programas de desarrollo, se enfrentan progresiva y sistemáticamente a la crítica de organizaciones que rechazan los principios y los impactos de las actividades extractivas, y b. Describir cómo los movimientos sociales y territoriales críticos recogen la cuestión de fondo e inducen la creación de nuevos modelos de desarrollo, emancipados de los cánones de la modernidad, la ciencia, Occidente y las estructuras hegemónicas del conocimiento y la vida social. La hipótesis central, es que ambos países viven en la actualidad procesos de crisis que denuncian la obsolescencia del modelo de desarrollo extractivo en América Latina (Göbel y Ulloa, 2014; López Navia, 2019), sea en su versión progresista y de transición postneoliberal, conservadora o neoliberal. Ambas versiones se enfrentan hoy a los múltiples cuestionamientos a su compromiso con la continuidad del paradigma extractivista, considerado por los movimientos sociales como incompatible con cualquier proyecto real de sustentabilidad. En otras palabras, tanto el Vivir Bien como el desarrollo sustentable, decantan hoy en propuestas que son objeto de críticas y cuestionamientos que ya han sido formulados previamente en el contexto de los paradigmas convencionales de desarrollo. Esta cuestión cobra especial relevancia en la actualidad, en que Bolivia vive una profunda crisis interna de su democracia y que en Chile el movimiento social del último año ha enfatizado la crítica a la premisa del desarrollo centrado en el crecimiento económico de base extractiva y transnacional. A partir de esta hipótesis, se avizora que América Latina puede estar enfrentando entonces, el inicio de un período de auspiciosos procesos de replanteamiento, donde las miradas respecto de aquello que ha resistido y sobrevivido a las representaciones dominantes de la economía y el desarrollo, pueden avanzar hacia adquirir un nuevo lugar político. Los métodos utilizados para el abordaje de esta discusión provienen del análisis de fuentes secundarias -bibliografía especializada y fuentes oficiales $-\mathrm{y}$ los datos de campo recopilados directamente en el examen empírico de procesos de desarrollo en Bolivia y Chile, por parte de las autoras. La organización y el análisis de los datos 
se llevó a cabo a partir de los principales aspectos expuestos en las preguntas e hipótesis propuesta. A partir de allí, el texto se organiza en dos partes, directamente concordantes con estos aspectos centrales a la problemática planteada. Una primera, en que se desarrolla la presentación de los contextos de estudio, identificando actores y procesos en tramas políticas y culturales territorializadas. Posteriormente, se ofrece una descripción de los dos principales marcos ideológicos culturales que organizan al desarrollo en ambos países: el Vivir Bien en el caso de Bolivia y el desarrollo sustentable en el caso de Chile. En una segunda parte, el texto se centra en la cuestión del extractivismo, ilustrando el modo en que se expresa en cada caso, y las reacciones que este ha suscitado desde comunidades locales y movimientos sociales en defensa de los territorios. A partir de estas reacciones se configura en el texto el argumento empírico de las actuales crisis del desarrollo en América Latina, tanto en sus transiciones hacia un postneoliberalismo (Mun, 2015) como en el apogeo mismo del neoliberalismo transnacional (Pinol, 2015). Finalmente, el artículo concluye en torno a los actuales desafíos de replanteamiento y transición hacia otros modos de desarrollo en América Latina, basados en los reencuentros entre sociedades y naturalezas, hacia la construcción de nuevas normalidades.

\section{Contexto Chile: el advenimiento del desarrollo sustentable}

A partir de la década de 1990, el Estado de Chile comienza a experimentar la diversificación de sus materias de gestión, en concordancia con los procesos globales y los acuerdos internacionales que así lo requerían. En un contexto marcado por la ilusión que para una gran mayoría significó el retorno a la democracia, el país se enfrentó a la creación de nuevos espacios administrativos en torno a temas que trajeron consigo nuevas miradas a los procesos de desarrollo en el país. Tras diecisiete años de dictadura (1973-1990), no obstante, el conglomerado de oposición a la derecha y al gobierno encabezado por los militares, no promueve cambios en las estructuras de la economía que desde el golpe de Estado venían regulando al país. En palabras de Angell (2009): "había ahora un acuerdo mucho mayor respecto a las políticas económicas: casi todos los Partidos aceptaban que el mercado y el sector privado tenían un rol fundamental en el desarrollo económico" (p. 251). Desde este prisma, y sin ánimos de robustecer -aunque sí de modernizar- el rol del Estado en el bienestar, el país se enfrenta al desafío heredado de mantener las proyecciones del crecimiento económico de acuerdo con las propuestas neoliberales y a hacer frente a las principales deficiencias en materia de pobreza y desigualdad social. Los primeros años del gobierno de Patricio Aylwin propiciaron la implementación de nuevas agendas programáticas para la gestión pública, abriéndose gradualmente a las condicionantes políticas de los mercados internacionales y a los acuerdos interestatales en materias políticas y económicas. Eran los tiempos de rearticulación del país con un nuevo escenario global, para ello se hizo uso de estrategias tales como la de exhibir un iceberg en la Exposición Universal de Sevilla en 1992, como evidencia de una imagen basada en ideas tales como: Chile empresa de ideas, Chile funciona, País de riqueza generosa, Gente sólida (Correa et al., 2001) ${ }^{1}$. Simultáneamente, los primeros gobiernos de la coalición denominada Concertación de Partidos por la Democracia, trabajaron durante las décadas siguientes en la creación de institucionalidades, roles y normas asociadas a los nuevos temas de la agenda internacional: la cultura, las mujeres, los pueblos indígenas, los jóvenes y el medio ambiente, entre otros.

En este contexto, la nueva gestión medioambiental trajo consigo el uso de la idea de sustentabilidad mediante la Ley N ${ }^{\circ} 19.300$ Sobre Bases Generales del Medio Ambiente, que se aplica en Chile desde 1994. En este marco legal, el desarrollo sustentable es definido en su Artículo 2 letra g, como "el proceso de mejoramiento sostenido y equitativo de la calidad de vida de las personas, fundado en medidas apropiadas de conservación y protección del medio ambiente, de manera de no comprometer las expectativas de las generaciones futuras". De este modo, el país y la gestión pública comienza a armonizarse con los lineamientos globales promovidos desde la década de los 80 a partir de la publicación del Informe Brundtland, reafirmados en la Conferencia de las Naciones Unidas sobre el Medio Ambiente y Desarrollo realizada en Río de Janeiro, Brasil, en 1992. En paralelo a esta innovación conceptual y de gestión, el sector privado inicia también un lento proceso de adopción de la idea de sustentabilidad -que como veremos luego, se socializa y masifica a nivel sociocultural de la mano de nuevas carreras universitarias, nuevas institucionalidades, 
nuevas áreas de gestión, asociados a temas tales como los recursos naturales renovables, la gestión ambiental, el control de impactos de la producción-. Todas estas transformaciones van de la mano de un advenimiento global de la cuestión ambiental, que parece dar cuenta de un nuevo revestimiento ético y político para el desarrollo económico a nivel internacional. Desde esta economía ambiental se propone el control de las externalidades sociales y ambientales de la producción extractiva, ante ello, se reafirma la confianza en las mediciones y diseños de gestión de base científica como base de la gestión pública y privada. Los objetivos de intensificar la participación del país en la economía internacional se mantienen robustos, por lo que los modelos de gestión del desarrollo se actualizaron, sofisticando lenguajes y prácticas ahora enmarcadas en esta idea de "desarrollo sustentable".

Desde el punto de vista antropológico, ha sido posible observar las maneras en que estas transformaciones se han ido desplegando en los territorios, generando nuevos escenarios y órdenes de relaciones económicas y sociales. La apertura de los mercados internacionales en tanto fenómeno macroeconómico, adquiere allí su dimensión sociocultural territorial y localizada. La vivencia de la transnacionalidad en los territorios, se expresa en su vida cotidiana, y, en el caso de Chile, fuertemente marcada por las nuevas directrices "sustentables" que hegemónicamente debe llevar la producción y la gestión. En territorios con presencia extensiva de industrias extractivas -mineras, forestales, agroindustriales, entre otras- es posible observar cómo estas industrias conducen la construcción de los espacios públicos, las circunstancias de las economías locales y sus índices de desarrollo, la definición de liderazgos y formas de organización, entre otros dominios sociales e intersubjetivos que hacen forma y sentido de la vida social. En efecto, el peso de los grandes capitales y sus modos de vida en territorios con historias productivas y culturales previas, supone una transformación permanente, ejercicios y prácticas constantes de resistencia, adaptación, integración (Pino y Carrasco, 2019).

Pero más allá de los desequilibrios visualizados desde las ópticas de la dependencia, los procesos recientes en los territorios del centro sur de Chile evidencian desigualdad y complejidad, propios de sociedades que viven y sobreviven a la expansión capitalista. Allí, los mundos locales e indígenas han sido redefinidos a partir de las nuevas condiciones que los mercados internacionales imponen a las grandes empresas, en materias de impactos ambiental y social. Esto ha concedido en el escenario neoliberal chileno, una entrada ancha y contundente para las empresas en el diseño y la implementación de los procesos de desarrollo en los territorios, los que se han visto condicionados en su biopolítica a la reproducción de ideas dominantes asociadas a la educación, las tecnologías, la infraestructura, la organización social (González Hidalgo, 2018). En estos contextos, en que se vive la reproducción de estas construcciones dominantes del desarrollo basadas en la centralidad del capital, simultáneamente se gestan y consolidan iniciativas críticas, reivindicaciones de autonomía y expresiones alternativas. Visto desde esta perspectiva, el capitalismo no solo es cuestionado en y desde los territorios, sino más aún, relativizado por medio de las denuncias de comunidades locales articuladas para insistir en el establecimiento de límites para el crecimiento económico. Todo esto, resurge en la actualidad en el marco de los complejos procesos de crisis social que afectan al país, donde la fuerza del llamado a un nuevo pacto social da cuenta de un fuerte descontento con el modo de vida neoliberal (Bassa, 2020).

En la experiencia de actores, comunidades locales y territorios, se vivía ya en la década de los 90 el advenimiento de una crisis ecológica, derivada del protagonismo de la economía formal en la toma de decisiones y de la ausencia de nuevas visiones de desarrollo que integrarán de manera efectiva "los saberes subyugados y la complejidad del mundo negados por la racionalidad mecanicista, simplificadora, unidimensional, fraccionadora que ha conducido el proceso de modernización" (Leff, 2004: 18). En las últimas décadas, organizaciones territoriales y movimientos sociales de escalas locales y globales han ido demostrando mediante diversos mecanismos, su incomodidad y crítica a la expansión de la economía a todos los dominios de la vida. Esto detona incesantes procesos territoriales de conflicto y negociación permanente, donde se transan de modo sistemático las concepciones de desarrollo y de naturaleza, cuestión especialmente delicada en el caso de territorios marcados por industrias extractivas y presencia de pueblos originarios.

Siguiendo esta mirada crítica, el desarrollo sustentable o el discurso de la sustentabilidad implementado oficialmente en Chile por parte del Estado por medio de su política ambiental y sus políticas 
sectoriales de desarrollo productivo, prioriza el resguardo de las condiciones para el crecimiento económico, y no la transformación de dicho crecimiento en virtud de las condiciones ecológicas y sociales que lo solventan. En lo que respecta al sector privado, el mundo empresarial que ha controlado al país en materias económicas, políticas y culturales desde el golpe de Estado, también ha debido enfrentarse a la reforma propiciada por los requerimientos de la sustentabilidad. No obstante, su adopción no se debe a que el Estado se lo exija por medio de normas y procesos de fiscalización eficientes, sino más bien por los giros que los mercados internacionales han venido dando en torno a las condiciones de la producción. Este aspecto es descrito en el apartado siguiente, usando como principal referencia lo acontecido en Chile con el paradigma del manejo forestal sustentable.

\section{Contexto Bolivia: La constitucionalización del Vivir Bien y su construcción como proyecto político-económico}

Teniendo sus raíces en la cosmovisión indígena de los quechuas y aymaras, el suma qamaña o suma kawsay (vivir bien) es un concepto andino originado en filosofías ancestrales que aspira a una vida cosmocéntrica, donde se relaciona la Naturaleza y la comunidad humana de forma armónica, sin fines de egoísmo, consumo e individualismo (CañedoArgüelles, 2012: 111/112; Huanacuni, 2010: 21). De tal manera, un eje fundamental en el concepto original del Vivir Bien está formulado con una crítica hacia el desarrollo capitalista y la priorización del crecimiento económico (Villalba-Eguiluz y Etxano, 2017: 2). Reconociendo la crisis global del capitalismo con sus correspondientes impactos sociales y ambientales, el Vivir Bien adquirió atención internacional siendo una alternativa que sustituye los conceptos convencionales del desarrollo desde lógicas puramente económicas por factores como la interculturalidad, la concepción comunitaria y armónica con la naturaleza y el respeto por otras formas de vida y sus espacios (Mamani Pacasi $e t$ al., 2012: 31).

La constitucionalización del concepto de "Vivir Bien" en Bolivia estuvo marcada por un contexto histórico global, donde una variedad de gobiernos del Sur global aspiraba a recuperar el rol activo del Estado y fortalecer la soberanía nacional para enfrentar las privatizaciones, masivas inversiones extranjeras y, sobre todo, la apertura de las economías a los mercados globales (Aroca Ribadeneira, 2020; Lander, 2011: 21/130).

Mediante el proceso de cambio iniciado en 2005, en cuya base se articuló la Nueva Constitución Política del Estado Boliviano de 2009, el Vivir Bien logró articularse en Bolivia como proyecto político del gobierno del MAS-IPSP ${ }^{2}$. Ese proceso de cambio, encabezado por el primer presidente indígena en Latinoamérica, Evo Morales, tuvo como objeto fundamental una ruptura con un Estado oligárquico y colonial central, permitiendo rescatar la influencia de ideas descolonizadoras y antineoliberales y promover los valores de "justicia, equidad y dignidad" (Programa del Gobierno del MAS-IPSP, 2010-2015). El Vivir Bien fue construido en este contexto propiciado por el proceso de cambio, y por tanto, fue concebido como un proyecto político en el marco de la búsqueda de un nuevo modelo económico principalmente opuesto al neoliberalismo e imperialismo, para garantizar el bienestar de toda la población. Esto demuestra que el Vivir Bien en Bolivia, similar al caso ecuatoriano, se convirtió un paradigma en construcción, que experimentó varios procesos de resignificación, siendo el contexto político uno de estos (Aroca Ribadeneira, 2020). La definición fundamental del Vivir Bien en Bolivia se introdujo mediante el Artículo 8 de la Constitución del Estado de $2009^{3}$, donde se le presentó en el gobierno de Evo Morales como nuevo modelo para el desarrollo en relación con las bases fundamentales del Estado (Gudynas, 2011: 233; Puente, 2011: 345/346). De todas formas, es sumamente importante destacar que la traducción del Vivir Bien por parte del gobierno del MAS-IPSP en el proceso de cambio y el contexto socioeconómico de Bolivia trajeron nuevos horizontes a este modelo de desarrollo, que se alejan marcadamente de sus conceptos originales. Estos nuevos horizontes fueron condicionados por el contexto de la lógica nacional-popular en la Constitución Política del Estado de 2009 y las raíces del MAS-IPSP, que se originan en la conjunción de una diversidad amplia de corrientes y actores sin una ideología unitaria. Como se pudo comprobar en un estudio empírico llevado a cabo en el 2013, las construcciones subjetivas del sector dominante del MAS-IPSP acerca del Vivir Bien están vinculadas con el objetivo central de generar una satisfacción de las necesidades básicas por medio de los elementos de salud, educación e infraestructura, para lograr un incremento de la 
calidad de vida para la población boliviana (Lehnert, 2013). De tal manera, el Vivir Bien fue interpretado como una meta para el anterior gobierno de Evo Morales, que se debería alcanzar para la población boliviana mediante programas sociales que aspiran a la satisfacción de las necesidades básicas de esta. Este objetivo central está sujeto, como los mismos dirigentes del MAS-IPSP declararon, a la necesidad de maximizar las ganancias económicas y generar mayores excedentes mediante el rol activo del Estado. No obstante, aunque fuese recuperado el control del Estado en la industria de hidrocarburos y minería mediante las nacionalizaciones, no se impusieron políticas de expropiación de los bienes de los operadores transnacionales (Andreucci y Radhuber, 2017; Ceppi, 2016: 185). El objetivo central de obtener un mayor control de la renta petrolera fue acompañado más bien por una continuación de la inversión extranjera, la que llevó a una expansión de las actividades extractivistas (Andreucci y Radhuber, 2017; Ceppi, 2016: 185). En consecuencia, Ceppi (2016) caracteriza la política económica del gobierno del MAS-IPSP Bolivia por una "convivencia entre el nacionalismo extractivista y la inversión extranjera". Estas dinámicas no solamente se observan en el sector de hidrocarburos, sino también en el del litio, donde se cuestiona la recuperación estatal de los recursos naturales como lo fue prometido con las reformas del proceso de cambio (Campanini, 2019; Fundación Solón, 2019). La recuperación del control de la industria de hidrocarburos fue un factor central que el gobierno de Evo Morales consideró para el desarrollo del sector primario exportador como fuente de ingresos necesarios para instaurar las políticas de redistribución concordante con el Vivir Bien. Además, desde el punto de vista del escenario internacional, los altos precios de las materias primas y la demanda china, ofrecieron incentivos para la continuación de este modelo económico basado en la extracción de materias primas (Villalba-Eguiluz y Etxano, 2017: 6).

Este contexto político-económico del Vivir Bien ilustra claramente que, mientras la concepción original ancestral de los indígenas respecto del Vivir Bien contiene principios anticapitalistas y prioriza la vida armónica con la naturaleza, la interpretación ideológico-política del sector principal del MAS-IPSP acerca del Vivir Bien no modifica las racionalidades y estructuras productivas de la economía internacional. Esto se reafirma además por la ausencia de reformas estratégicas, entre ellas la fiscal. Desde esta perspectiva, la construcción del Vivir Bien como proyecto político-económico si bien pudo favorecer la recuperación de sentidos políticos e identitarios de los pueblos originarios y movimientos sociales que ahora forman parte del Estado plurinacional, también reafirmó la alta dependencia del mercado internacional de recursos naturales, a partir de ello el Estado daba pasado a una apuesta neoextractivista (Svampa, 2016). Las lecturas nacional-populares y modernizantes del anterior gobierno boliviano de Evo Morales referidas al Vivir Bien, por tanto, no llevaron a una perspectiva transformadora del modelo de desarrollo propiamente tal. En la medida que el gobierno se siguió abriendo al comercio internacional con los hidrocarburos y otorgó al mercado global un rol central en la asignación de los recursos, su visión intervencionista quedó simultáneamente vinculada con la reproducción del paradigma convencional de desarrollo basado en actividades extractivas a gran escala.

En concordancia con los procesos económicos en el desarrollo sustentable implementado en Chile, la lectura del Vivir Bien por parte del MAS-IPSP en Bolivia aspiró a que se crearan las condiciones para el crecimiento económico para poder asegurar el "Vivir Bien", por ello el rol del mercado internacional y las actividades extractivas siguen siendo sumamente relevantes. No obstante, en ambos casos se evidencia la reformulación de los modelos de gestión del desarrollo económico. Mientras que en Bolivia el Estado por medio de la Constitución de 2009 induce a un actuar gubernamental que reconoce a empresas y a actores comunitarios como parte de un modelo económico basado en la recuperación de los recursos naturales (Bazurco, 2020), en el caso chileno se resguardan las condiciones para el crecimiento económico mediante la potencia del sector privado y el actuar de las empresas transnacionales en los territorios. Es decir, se deposita en las grandes empresas la responsabilidad de apoyar el desarrollo económico de los territorios, dando paso con ello a un profundo y sistemático proceso de colonización cultural que radicaliza la vigencia en Chile del sistema sociocultural capitalista. Desde esta racionalidad, se diseñan e implementan compensaciones y convenios con comunidades indígenas y locales afectadas por los impactos sociales y ambientales de la producción, acciones que buscan enmarcarse dentro del modelo de desarrollo sustentable en temas 
como el desempleo, la pobreza y la conservación de la biodiversidad.

En cuanto a los tipos de impactos sociales y ambientales de las actividades extractivas en territorios específicos, Bolivia y Chile presentan grandes similitudes. Con las prácticas extractivistas, que ahora están enmarcadas por el concepto del "Vivir Bien", en Bolivia también se evidencian desequilibrios y complejos escenarios de crisis. Las bases de estas crisis se encuentran en la prevalencia en ambos países del paradigma dominante de desarrollo basado en una economía primario-exportadora que, cada vez con mayor complejidad, entra en pugna con concepciones locales de desarrollo y territorialidad.

\section{Formas y contenidos del extractivismo en Chile y Bolivia}

\section{Chile: el caso del extractivismo forestal}

En la actualidad existe en Chile un total aproximado de 3,5 millones de hectáreas plantadas principalmente con las variedades Pinus radiata y Eucalyptus globulus, las que marcan de manera determinante el paisaje entre las regiones de Valparaíso y Aysén. Dos tercios de esta cantidad pertenecen a grandes empresas forestales que administran desde la capital del país y centros urbanos de provincia, el ciclo productivo de estas plantaciones y su convivencia con familias y comunidades locales. La matriz productiva del monocultivo coexiste así con economías campesinas diversas, entre estas es posible encontrar desde medianos propietarios con desarrollo de emprendimientos para agregar valor a sus productos, nuevos habitantes rurales que desarrollan economías familiares con algo de producción agrícola y emprendimientos productivos y de turismo, pequeños propietarios que desarrollan economías de subsistencia con escasa vinculación con el mercado y familias sin tierra que viven en la propiedad de familiares formando parte de economías de subsistencia y acrecentando las cifras de pobreza rural ${ }^{4}$. Se trata entonces de dinámicas de coexistencia entre formas económicas y socioculturales que se hacen complejas en el territorio que comprende entre la provincia de Arauco en la región del Biobío y la región de Los Lagos, en que el pueblo mapuche reivindica la presencia histórica y ancestral del Ngulumapu (Nahuelpan, 2013). La configuración del territorio se torna así densa e intensa, debido a la convivencia friccionada entre las expresiones económicas extractivas de gran escala representada por las empresas forestales, y las propuestas de autonomía indígena basadas en concepciones sagradas de la naturaleza y sociales de la economía. En estos territorios circulan por tanto diversas formas de comprender a los recursos naturales y la administración de la vida material o economía, lo que supone la existencia de tensiones y conflictos latentes y manifiestos en torno a estos recursos y sus usos.

Como evidencia de esta complejidad, en estudio realizado en $2015^{5}$ fue posible identificar seis ámbitos críticos que revelan la naturaleza problemática de esta convivencia. Aun cuando existen posiciones favorables y acríticas en cuanto a las implicancias de la presencia de los monocultivos forestales -especialmente en territorios de las regiones de O'Higgins y Maule-, lo cierto es que se trata de un patrón productivo y cultural controversial, que ocasiona importantes incomodidades y dificultades para otros modos de vida. Los principales ámbitos críticos de esta convivencia que han sido identificados son: las relaciones entre empresas y comunidades locales, la propiedad de la tierra, los impactos operacionales, los aspectos socioambientales y el desarrollo económico local (Informe Consultoría V 26: 10). En cada uno de estos ámbitos se expresa un conjunto específico de problemas, entre los que se destacan: a) los asociados a la convivencia directa y las relaciones cara a cara entre habitantes y representantes de las empresas forestales, b) la crisis hídrica que se adjudica -entre otras razones- a la expansión progresiva y sistemática de las plantaciones forestales y al incumplimiento sistemático de normas asociadas a las distancias entre estas y las fuentes de agua, c) la desaparición progresiva de la agricultura producto de la expansión de los monocultivos y la desnutrición y desertificación de los suelos y d) las limitaciones que las plantaciones forestales imponen para el desarrollo de otro tipo de actividades productivas y escalas económicas.

Las denuncias de comunidades y organizaciones locales son sistemáticas. Las situaciones críticas son frecuentemente difundidas por las plataformas de movimientos sociales y medios de comunicación afines ${ }^{6}$. Comunidades locales sin acceso a agua, familias afectadas por operaciones forestales, dificultades para establecer acuerdos con las empresas, e incluso la opción de acusar al Estado por el enriquecimiento del patrimonio de grupos económicos a costa de "la destrucción de 
estos territorios" (Arriagada, 2017), forman parte de la agenda de la resistencia a la expansión de las plantaciones forestales en Chile. Claramente, para estos habitantes de los territorios, la convivencia con los monocultivos resulta ser una condición negativa. Es una convivencia que se traduce en la asfixia de sus propios modos económicos y sistemas productivos, basados en tramas de relaciones sociales que hoy se ven abiertamente fracturadas por el despoblamiento rural. Frente a ellos, la definición de la producción forestal como una actividad sustentable resulta una ironía, no obstante, tanto el Estado como el mercado global así lo vienen definiendo en el marco de las propuestas de Política Forestal, 2015-2035 y los sistemas internacionales de Certificación de Manejo Forestal, respectivamente. Ambas herramientas, conciben implícitamente a las plantaciones de monocultivos a gran escala como una actividad que puede convivir con otros sistemas económicos y culturales, cuyos impactos sociales y ambientales pueden ser gestionados. Las ciencias que solventan a este paradigma reafirman también la importancia de "incluir" a las comunidades locales en el desarrollo económico, de reducir los impactos operacionales (Organización de las Naciones Unidas para la Alimentación y la Agricultura, 2016) y de respetar los derechos indígenas (siguiendo las indicaciones del Convenio 169 de la Organización Internacional del Trabajo sobre Pueblos Indígenas y tribales). No obstante, las resistencias y reacciones críticas no cesan en demostrar que esto no es posible debido a lo que comprendemos como las incompatibilidades ontológicas entre la racionalidad y el sistema sociocultural empresarial transnacional y las concepciones de naturaleza, economía y desarrollo reivindicadas por la territorialidad ancestral del pueblo mapuche.

Lo anterior, deja en evidencia tanto los límites de la comprensión global e institucionalizada de la sustentabilidad transnacional como también la importancia de revisar las cualidades de este tipo de actividad forestal comprendida como una actividad extractiva. De acuerdo con las definiciones con mayor consenso, estas refieren a aquellas actividades basadas en la explotación de recursos naturales, a gran escala, con bajo nivel de procesamiento y dirigidas a mercados de exportación. En términos estructurales, este tipo de actividades serían la base de las economías latinoamericanas del último siglo, propiciando la participación de estas en los mercados internacionales, pero al mismo tiempo, la crisis de sus nuevas apuestas conceptuales, específicamente en el caso chileno, lo que interpretamos como los límites y la crisis del discurso global del desarrollo sustentable. A partir de los datos disponibles es posible sostener que las plantaciones de monocultivos forestales a gran escala constituyen una actividad extractiva debido a que cumplen con los criterios consensuados antes descritos, pero también por tratarse de una matriz productiva y de desarrollo económico y cultural expansivo. Bajo esta condición, el denominado "manejo forestal sustentable" da cuenta de las cualidades contemporáneas del capitalismo global que hoy da sentido a los discursos, lenguajes y prácticas oficiales y hegemónicas de la sustentabilidad en Chile. Desde esta perspectiva se intensifican hoy los procesos de control sociocultural y de agencia creativa que ejerce el capitalismo corporativo de las grandes empresas forestales en territorios múltiples y desiguales. Aquí se encontraría, desde una perspectiva crítica, el principal meollo de las crisis políticas y sociales actualmente determinantes de la realidad chilena, derivada del agotamiento de los sistemas de relaciones sociales en territorios cuya población habita, participa, sobrevive, confronta, cuestiona y propone, simultáneamente.

\section{Bolivia: el extractivismo en la región del Tipnis}

El Tipnis ${ }^{7}$ es un parque nacional y territorio indígena que se ubica entre los departamentos de Beni y Cochabamba y limita al este con la selva amazónica. Disponiendo de una gran cantidad de suministros de petróleo y gas, la región del Tipnis se transformó en espacio de disputa con el proyecto de carretera Villa Tunari-San Ignacio de Moxos, del gobierno de Evo Morales, para facilitar la extracción de las materias primas (Hope, 2016: 925; Lalander, 2016: 11). El proyecto de carretera provocó un conflicto amplio que logró atención nacional e internacional a partir del 2010. La decisión de retomar en 2009 planes antiguos para la construcción de una carretera nacional, que debía conectar la tierra baja del Amazonas con el altiplano, fue tomada por el gobierno de Evo Morales sin consulta previa con las comunidades locales que viven en la región (Hope, 2016: 925). El 15 de agosto de 2011 los pueblos indígenas (pueblos mojeño, yuracaré y chimán) del Territorio Indígena Parque Nacional Isiboro Sécure iniciaron una marcha de más de 600 kilómetros hacia la ciudad de La Paz en rechazo a la construcción de 
la carretera. Por su parte, los indígenas del oriente rechazan principalmente el tramo 2 (Isinuta-Monte Grande), ya que esta parte de la carretera pasa directamente por el área protegida del Tipnis. El gobierno del MAS-IPSP justificó la construcción de la carretera con el discurso del Vivir Bien, porque el mejoramiento de la infraestructura de la región facilita la extracción de materias primas y la apertura al mercado global, lo que son condiciones fundamentales para generar mayores excedentes destinados a las políticas públicas. El conflicto respecto de la construcción de la carretera en el Tipnis se torna así representativo para los límites del Vivir Bien, porque los objetivos del gobierno de una justicia redistributiva y reformas de bienestar chocan con los derechos de los pueblos originarios y de la Madre Tierra en el territorio, e impiden una conciliación entre los distintos modos de desarrollo de los actores involucrados (Lalander, 2016: 1). Considerando los beneficios que resultan de la construcción de la carretera para los convenios y empresas transnacionales, se identifica con mayor profundidad el rol del capitalismo global en este conflicto. Conjuntamente con la facilitación de la apertura al mercado asiático para la exportación boliviana y brasileña, son los megaproyectos internacionales tales como el "Plan Iniciativa Regional Sudamericana" (IIRSA) con Brasil los que se benefician significativamente del proyecto de la construcción de la carretera (Paz, 2011: 25). Estas dinámicas económicas evidencian que el mercado global es un factor clave que condiciona el paradigma de desarrollo acerca del Vivir Bien en el gobierno del MAS-IPSP, que determina la biopolítica de los territorios que se mantienen de este modo hipotecados a la economía extractiva y centrada en el capital.

La inscripción de las dinámicas económicas capitalistas en el Vivir Bien, desdibujan así sus impactos como modelo de desarrollo alternativo y justifica el extractivismo. Muy similar al caso chileno del extractivismo forestal, el conflicto de la carretera en la región del Tipnis desvela la reproducción de dinámicas territoriales complejas. Allí, el discurso hegemónico del gobierno acerca del Vivir Bien no favorece la coexistencia entre distintos modos de vida y provoca tensiones y resistencias, priorizando los intereses del capital por sobre otros criterios de desarrollo.

La diversidad de la región del Tipnis se demuestra por sus habitantes: los pueblos mojeño, yuracaré y chimán y los campesinos cocaleros, todos diferentes actores con distintos fondos económicos y socioculturales. Con el proyecto de la carretera se introdujo además la visión dominante de desarrollo del Estado boliviano acerca del Vivir Bien y se hicieron complejas las problemáticas ya existentes entre los pueblos originarios y los cocaleros. Desde mediados de la década de 1960, cuando familias campesinas, principalmente aymaras y quechuaparlantes se dirigieron de Cochabamba a la zona protegida para la agricultura de coca, los pueblos originarios del Tipnis se vieron confrontados con la expansiva colonización de los cocaleros. Mientras que el modo de desarrollo de los colonizadores andinos consiste en la comercialización de la hoja de coca donde se aprovechan los bienes del bosque de forma individual y se abstiene de conceptos como la convivencia y reciprocidad con la naturaleza y el ser humano, los pueblos indígenas yuracarés, chimanes y moxeños se caracterizan por un modelo de propiedad colectiva donde se aspira a una actividad agrícola de subsistencia (Paz, 2012: 4). El proyecto de construcción de la carretera en el Tipnis por tanto no solamente fue importante para el Estado y los mercados internacionales, sino también para los campesinos cocaleros de la región, porque el mejoramiento de la infraestructura de la región implica la expansión de los cultivos de coca y su industrialización.

La complejidad del conflicto en la región del Tipnis, asociada a la diversidad de actores e intereses involucrados (diferentes comunidades locales, Estado, mercado internacional), devela las problemáticas del nuevo concepto político del "Vivir Bien" en su relación con el paradigma convencional de desarrollo. La lucha entre las distintas formas de producir, la propiedad privada de los cocaleros colonizadores contra la propiedad comunitaria de los indígenas, la continuación de la importancia del extractivismo y de los mercados internacionales y el subsidio del Estado demuestran que con una construcción dominante de desarrollo, que se basa en la reproducción de las estructuras económicas y culturales convencionales, es difícil establecer una forma de coexistencia solidaria y justa entre estos principales promotores. De modo similar a los límites del discurso transnacional del desarrollo sustentable en territorios mapuches en Chile, la apuesta del Vivir Bien en Bolivia conlleva desequilibrios ontológicos a la región del Tipnis. El capitalismo global en ambos casos, tensiona la convivencia en la diversidad dentro de los territorios 
por medio de la imposición de un diseño hegemónico del desarrollo.

\section{Reacciones críticas. Los planteamientos de organizaciones y movimientos sociales en contra del desarrollo sustentable en Chile y del Vivir Bien en Bolivia}

\section{Chile: ¿manejo forestal sustentable?}

Como se indicó previamente, la construcción de los discursos y las prácticas hegemónicas del desarrollo sustentable en materia forestal en Chile provienen tanto de la política nacional como de los sistemas de certificación internacional. Estos últimos han marcado los discursos y las prácticas empresariales de las últimas décadas, debido a que se han convertido en cuerpos de normas que

\begin{abstract}
"revisan y controlan diversos aspectos del manejo y producción forestal, promoviendo la adecuación de prácticas productivas, ambientales y sociales en sintonía con los requisitos de competitividad de los mercados internacionales cuya demanda se organiza hoy desde los mismos principios que subyacen a los instrumentos de certificación forestal" (Carrasco, 2015: 92).
\end{abstract}

Los sistemas con mayor presencia en Chile son el Pan-European Forestry Certification (PEFC) ${ }^{8}$, International Standard Organization (ISO) 14.000, y el Forest Stewardship Council (FSC). Estos sistemas han implicado el surgimiento de "un nuevo momento en la organización política de la gestión forestal del país (...) que cruza desafíos económicos y comerciales -de orden global, con desafíos políticos, sociales y ambientales localesde orden nacional y local" (Carrasco, 2015: 93) En su aplicación, y bajo la supervisión permanente de las empresas que evalúan por medio de auditorías anuales a las empresas certificadas, estos sistemas han logrado reorganizar los lenguajes y procesos que determinan la presencia de los monocultivos en los territorios. Es decir, han propiciado un nuevo momento en el ejercicio del poder corporativo, asociado ahora a la promoción del desarrollo desde los criterios hegemónicos de la sustentabilidad. Este nuevo momento ha implicado transformaciones organizacionales al interior de las empresas forestales, el uso de lenguajes renovados por parte de sus ejecutivos y agentes profesionales, y la inclusión de tópicos como los derechos indígenas, la conservación de la biodiversidad, y el desarrollo económico local en la gestión corporativa. Las empresas se han ido transformando así en actores con incidencia directa en la conducción y materialización del desarrollo en los territorios, induciendo la empresarización, el emprendimiento y el desarrollo basado en la innovación y en la participación en los mercados mediante la formalización de las actividades de productores individuales y asociados. Otra evidencia de estos cambios performáticos de las empresas en los territorios, propiciados por los estándares internacionales de manejo forestal sustentable, se encuentra en el uso hoy cotidiano de la categoría de áreas de alto valor de conservación -que pueden ser espacios sagrados, identificados y reivindicados por comunidades del pueblo mapuche-. Este uso parece indicar que el mercado identifica las zonas sensibles para su expansión y fuerza a que las empresas aseguren el control de las mismas. En otras palabras, se trata de áreas reivindicadas desde ontologías sagradas de la vida y la naturaleza que por medio de estas estrategias del Manejo Forestal Sustentable quedan reducidas a áreas sujetas a planes de manejo, acciones de conservación y monitoreo controlado por las empresas.

Estos sistemas de certificación constituyeron durante su primer período de implementación -período en que las empresas forestales se preparaban y enfrentaban las primeras auditorías- una esperanza efectiva para agentes y organizaciones que promovieron su adopción por parte de las empresas forestales por considerarlos un potencial sistema de control social. No obstante, a partir de la obtención del certificado FSC por parte de las dos empresas con mayor patrimonio en el país, estas fuerzas promotoras se fueron volviendo críticas, pues a pesar de todas las deficiencias cuestionadas en su gestión, ambas obtienen entre el 2012 y 2013 el sello por Manejo Forestal Sustentable (y lo conservan hasta la actualidad).

La reacción de organizaciones, actores y comunidades locales que resienten críticamente su convivencia con los monocultivos forestales, frente a la obtención de este certificado por parte de las grandes empresas, fue la del rechazo. A partir de este momento, estas organizaciones toman distancia de los sistemas globales de certificación forestal, por considerarlos controlados por las 
propias empresas, aun cuando estos siguen siendo en estricto rigor, mecanismos de la sociedad civil.

En la actualidad, el panorama de las críticas al desarrollo sustentable promovido por el Estado en Chile y materializado por programas públicos y la acción de grandes empresas en los territorios, se ha vuelto cada vez más transversal. Redes sociales como facebook e instagram han facilitado notablemente la masificación de denuncias y la difusión de redes políticas entre actores de los territorios, organizados para confrontar las bases y oponerse a las continuaciones de la expansión capitalista. Esta situación se ha hecho mucho más patente en tiempos de pandemia por Covid 19, en que la población se mantiene con mayor dedicación a la comunicación virtual, en un escenario además expectante por el proceso constitucional que se inicia con las elecciones del 25 de octubre de 2020. Estas críticas son elaboradas y mediatizadas desde organizaciones que reivindican otros modos de relación y uso económico de los recursos naturales, que buscan fortalecer los vínculos comunitarios con los ecosistemas en perspectiva de gobernanza de los sistemas socioecológicos (Ostrom, 2009). Se suman a estos desarrollos críticos en torno a la sustentabilidad oficializada por el Estado y las políticas privadas en Chile, los trabajos de investigación elaborados desde enfoques también cuestionadores de la "Ciencia normal" y su supuesta objetividad ante procesos políticos y culturales inminentes. En este aspecto, destacan los desarrollos recientes de investigación en el campo de la ecología política, desde disciplinas como la arquitectura, la geografía, la antropología, el derecho, la economía, entre otras. Todos estos estudios comparten una perspectiva crítica de las bases y el quehacer de la ciencia convencional, y se suman, por tanto, a la crítica a los modelos dominantes de desarrollo centrados en la economía y el mercado. Avalan también la necesidad de visibilizar la diversidad de saberes, de poner a las ciencias en diálogo horizontal con otros conocimientos presentes en los territorios, potencialmente portadores de otras ideas de naturaleza y desarrollo. En síntesis, la importancia de perseverar en la ecología de saberes y avanzar hacia la justicia cognitiva desde el conocimiento científico contrahegemónico, por medio de la promoción de "la interdependencia entre conocimientos científicos y no científicos" (De Sousa, 2010: 45).

\section{Reacciones, movimientos y críticas al Vivir Bien en Bolivia}

La implementación de los procesos de desarrollo inspirados en el Vivir Bien en Bolivia, intensifican la complejidad de los territorios sujetos a los requisitos de movilidad y expansión de la economía internacional, ya que las posiciones y los intereses de los diferentes grupos de actores en protesta son diversos y múltiples. Las relaciones de poder están siendo reproducidas además por las empresas internacionales que inducen procesos transformadores en las percepciones de las comunidades locales.

Las resistencias de las comunidades locales del Tipnis organizadas colectivamente, difieren en su grado de oposición a las dimensiones progresistas subyacentes a las construcciones del Vivir Bien del gobierno de Evo Morales. Las protestas en contra de la carretera en la región fueron lideradas por la organización territorial "Subcentral Tipnis" y las confederaciones indígenas CIDOB $^{9}$ y CONAMAQ ${ }^{10}$, cuyo discurso dominante se refiere en primer lugar a la violación de la apropiación territorial (Hope, 2016: 925; Lalander, 2016: 10). Estas organizaciones no incluyeron en sus demandas oficiales una crítica explícita a la visión de desarrollo promovida por el Estado, ni se opusieron a las ideas desarrollistas de este. El gobierno perseveró en su visión de desarrollo centrado en el Vivir Bien con la argumentación que la carretera iba a traer beneficios económicos y crecimiento para la región del Tipnis (Hope, 2016: 926). Los manifestantes del Tipnis no rechazaron este argumento propiamente tal, pero sí cuestionaron los beneficios reales que la carretera pudiese traer para sus comunidades locales (Hope, 2016: 926). Siguiendo las argumentaciones de los dirigentes del Tipnis, las comunidades locales apoyan proyectos que aspiran al desarrollo de su región, mejorando la accesibilidad a salud y educación, pero rechazan el proyecto de la carretera del gobierno, porque no conecta las comunidades locales y pasa por la zona protegida (Hope, 2016: 926). Considerando las visiones de desarrollo de los pueblos del norte del Tipnis (los yuracarés, chimanes y mojeños) que defienden el territorio refiriéndose a su modelo socioeconómico sostenible, descrito como adaptativo a los ecosistemas y construido desde la propiedad colectiva, las diferencias entre las visiones de los modelos de desarrollo del Estado y de los indígenas 
se evidencian de forma más explícita. De esta forma, grupos indígenas locales cuestionan la justificación del extractivismo mediante el Vivir Bien por parte del gobierno boliviano, reivindicando las filosofías ancestrales del Vivir Bien para resguardar su vínculo con la tierra y su participación en los ecosistemas. Asimismo, las posiciones de los campesinos cocaleros que apoyan el proyecto de la carretera del gobierno extienden el grado de conflictos de intereses entre los diversos grupos. El contexto de diversidad cultural con diferentes actores y distintos fondos económicos y culturales juega entonces también un rol en las reacciones y movimientos críticos al Vivir Bien.

En el sector del extractivismo de litio en Bolivia la diversidad de perspectivas en las reacciones al desarrollo basado en el Vivir Bien es similar. Por un lado, las reacciones de las comunidades locales demuestran también la inducción de las ideas progresistas del gobierno y el control por las empresas internacionales para justificar el extractivismo. La investigación de Greenberg (2016) acerca de las perspectivas locales del litio en el Salar de Uyuni comprueba que para las comunidades rurales de Llica, Colcha K y Uyuni los proyectos para industrializar el litio en su región están relacionados significativamente con beneficios de progreso. Entre estos se reconocen el mejoramiento de la infraestructura, la creación de empleo o el aumento de ingresos económicos mediante las regalías. Pero en el estudio los entrevistados de las comunidades locales de la región también destacaron la falta de facilitación de información por parte del Estado boliviano acerca del proyecto de industrialización del litio en el Salar de Uyuni, lo que evidencia falta de participación de los territorios en el ejercicio de poder por parte del Estado y de las empresas internacionales. El alto nivel de pobreza de la región es otro factor por el que las comunidades locales apoyan proyectos que suponen un progreso económico para su región. Uyuni y Potosí, donde se encuentran los yacimientos de litio, se encuentran entre las regiones más pobres del país y del mundo. Por estas condiciones, el Comité Cívico Potosinista (Comcipo), las comunidades rurales y de los pueblos originarios se resisten de forma explícita a los proyectos de industrializar la producción de litio en el salar potosino de Uyuni. Cuestionan también la falta de entrega de información y de participación de Potosí y Uyuni en las ganancias del proyecto, por este motivo el
Comité Cívico Potosinista inició la anulación del contrato con la empresa alemana ACI Systems, el que fue retomado por el gobierno boliviano el 2 de noviembre de 2019, en medio de las crisis social que detonó en golpe de Estado.

Por su dependencia al ecosistema, los pueblos indígenas que practican la agricultura y ganadería alrededor del salar de Uyuni, están directamente afectados por los impactos ambientales de las actividades extractivistas (Campanini, 2019; Ströbele-Gregor, 2013). Las resistencias de estos actores se refieren al deterioro del medio ambiente, así como al cambio estructural que viven las comunidades rurales respecto de la forma de organizarse (Campanini, 2019; Ströbele-Gregor, 2013) y subsistir.

En el ámbito académico y de los movimientos sociales, las resistencias rompen con los discursos hegemónicos del Vivir Bien del Estado, cuestionando su vinculación con las estructuras económicas convencionales y la mencionada continuación de las actividades extractivistas. Los movimientos comparten en las redes sociales Facebook, Instagram y Twitter, principalmente, múltiples perspectivas críticas sistemáticas hacia el proyecto de la carretera en el Tipnis. En el mismo sentido que las resistencias a la expansión a las plantaciones forestales en Chile, y a múltiples conflictos socioambientales de la misma naturaleza en América Latina, informaciones y situaciones críticas son difundidas por medio de las plataformas, de maneras cada vez más fecundas y creativas. La difusión de la información acerca de las resistencias organizadas en las redes sociales, ayudaron al conflicto del Tipnis a adquirir relevancia internacional, debido a esto el caso del proyecto de la carretera fue llevado a la Corte Internacional de Justicia por los derechos de la naturaleza.

A las resistencias al proyecto de la carretera en el Tipnis se sumaron movimientos ambientalistas en las redes sociales, que criticaron la expansión capitalista en zonas protegidas por medio de la agroindustria y la ganadería. La inconsistencia entre las propuestas ecológicas y anticapitalistas de la versión original del Vivir Bien y la continuación de las actividades extractivistas del anterior gobierno forman parte central de estas críticas. Tanto en las redes sociales como en el ámbito académico, los argumentos en contra del proyecto de la carretera en el Tipnis y de la visión de desarrollo confabularon en una crítica mayor hacia la política del gobierno 
de Evo Morales y del MAS-IPSP. Esta oposición política se canalizó también en el contexto de las elecciones presidenciales del 20 de octubre de 2019, a partir de estas se detona el quiebre del orden democrático en Bolivia y, por tanto, la puesta en suspenso del proyecto constitucional del Vivir Bien.

\section{Conclusiones}

Ambos modelos, Vivir Bien en Bolivia y desarrollo sustentable en Chile, fueron parte de procesos de transformación política y cultural asociadas con procesos económicos globales, y en ambos casos se trató de paradigmas que coincidieron con la revolución de los medios y la globalización de internet y las redes sociales. Ambos paradigmas legitiman lo global, los extractivismos como bases de las economías nacionales y la participación en los mercados internacionales como indicadores centrales del desarrollo. Así también, ambos paradigmas asumen la continuación de una alta dependencia del mercado internacional.

Las dinámicas territoriales, por su parte, dan cuenta de nuevas y cada vez más densas complejidades en las relaciones de conflicto y negociación por el desarrollo, tanto en Bolivia como en Chile. Los diseños propiciados por los estándares del comercio y la certificación internacional, imprimen en los territorios nuevos paisajes y nuevos lenguajes, nuevos códigos para enfrentar la planificación del desarrollo, pero ajustados a los mismos cánones de siempre: infraestructura, tecnología, mercado. Frente a ello, los proyectos alternativos de desarrollo refrescan el debate y demuestran que el diseño hegemónico del territorio es solo uno más, y en ningún caso el único ni tampoco, necesariamente, el mejor.

El diseño de procesos de desarrollo basado en principios no extractivos ha sido una temática constante en las dinámicas territoriales de América Latina en las últimas décadas. En este contexto, han cobrado cada vez más importancia las posiciones divergentes que proponen otras fórmulas productivas y de relaciones económicas, basadas en principios postextractivistas. Estas apuestas están siendo construidas hoy por organizaciones territoriales y movimientos sociales, capaces de estructurar diseños comunitarios y regenerativos. En los mismos territorios en que se reproducen estos procesos, persevera la industria extractiva y el diseño dominante del desarrollo visualizado y vivido. Esta convivencia es entonces la dimensión clave de los actuales procesos de desarrollo en contextos rurales y de alta diversidad cultural, como los referidos en este trabajo respecto de Bolivia y Chile. No es una secuencia lineal de modelos que van siguiendo unos a otros, sino más bien una coexistencia llena de fricciones y cada vez más sofisticadas formas de poder.

Tomando en cuenta el rol hegemónico de las empresas transnacionales y la respuesta pasiva de los Estados ante las demandas por replantear el modelo de desarrollo, es posible comprender las crisis sociales en América Latina como crisis de este paradigma dominante.

Sin perder de vista las profundas diferencias entre un Vivir Bien inspirado en filosofías andinas y ancestrales y devenido en principio constitucional, y el desarrollo sustentable promovido estratégicamente para la regulación de los mercados internacionales, se reconoce en ambos casos la provocación de escenarios críticos donde las ontologías relacionales de la naturaleza y las comprensiones socioecológicas científicas antihegemónicas constituyen hoy los principales núcleos de resistencia.

Finalmente, los casos revisados muestran que para el debate de los procesos de desarrollo en Latinoamérica es fundamental mantener una perspectiva que tome en cuenta el análisis de las dinámicas capitalistas globales. Comprendiendo sus ritmos y criterios, se podrá a su vez interpretar las asimetrías vividas a escala territorial, como parte de relaciones de poder globales, donde sigue siendo el crecimiento del norte global, basado en el consumo de energía y recursos naturales, quien alimenta la necesidad de las actividades extractivistas en Latinoamérica (Lessenich, 2019). 


\section{Referencias Citadas}

Andreucci, D. y Radhuber, Isabella M.

2017 Limits to "counter-neoliberal reform": Mining expansion and the marginalisation of post-extractive forces in Evo Morales’ Bolivia. Revista Geoforum 84: 280-291.

Angell, A.

2009 Chile desde 1958. En "Chile desde la Independencia", editado por L. Bethell. Ediciones Universidad Católica Silva Henríquez, Cambridge University Press. Santiago.

Aroca Ribadeneira, K.

2020 Buen Vivir: Críticas y balances de un paradigma social en construcción. Revista Diálogo Andino 62: 41-55.

Arriagada, $\mathrm{J}$

2017 Entrevista con la dirigenta rural Jacqueline Arriagada en ' $\mathrm{el}$ Desconcierto.cl': Dirigenta rural analiza las consecuencias del DL 701: "La sequía de los territorios se convirtió en un negocio" (16 julio 2017), https://www.eldesconcierto.cl/2017/07/16/ dirigenta-rural-analiza-las-consecuencias-del-dl-701-lasequia-de-los-territorios-se-convirtio-en-un-negocio/ (2 septiembre, 2018).

Bassa, J.

2020 Chile Decide: Por una nueva Constitución. Santiago: Planeta.

Bazurco, M.

2020 Videoconferencia "Bolivia. Entre el Golpe de Estado y la pandemia", https://www.antropilles.org/ videoconferencia-bolivia-entre-el-golpe-de-estado-yla-pandemia-martin-bazurco-osorio-antropoleg/ (26 de septiembre de 2020)

Bebbington, A.

2009 Latin America: contesting extraction, producing geographies. Revista Singapore Tropical Geography 30 (1): 7-12.

Campanini, O.

2019 Das gute Leben, nur ein Diskurs- Interview mit Oscar Campanini über Extraktivismus für deutsche Elektroautos. Revista Lateinamerika Nachrichten (538), Berlin.

Cañedo-Argüelles, T.

2012 Diálogo cultural e intercambios como experiencia de Buen Vivir. La Suma Causai en Lima Norte. Revista Diálogo Andino 40: 111-129.

Carrasco Henríquez, N. y Salas Astrain, R.

2016 Examen crítico a la gestión capitalista de la diversidad cultural en el Manejo Forestal Sustentable. Un análisis del caso chileno. Política/Revista de Ciencia Política 54 (2): 153-175, doi: 10.5354/0716-1077.2016.44779.

Carrasco Henríquez, N. y Pino, A.

2019 Extractivismo forestal en la comuna de Arauco (Chile): internalización y formas de resistencia. Revista Colombiana de Sociología 42 (1), doi: 10.15446/rcs.v42n1.73233.

Carrasco Henríquez, N. y Salas Astrain, R.

2016 Inflexiones y dilemas ético-políticos del capitalismo en el Centro Sur de Chile. A propósito de la globalización forestal. Revista IZQUIERDAS, 27: 105-123.

Carrasco Henríquez, N.

2015 Pueblos indígenas y biodiversidad en el capitalismo contemporáneo. Propuesta para el estudio etnográfico de los procesos de certificación forestal en Chile. Revista Justiça do Direito qualis B4, 29 (1): 88-107, Universidade de Passo Fundo. Brasil.
Ceppi, N.

2016 Los hidrocarburos en el Gobierno de Evo Morales: extractivismo nacionalista con presencia extranjera. Revista Civilizar: Ciencias Sociales y Humanas 16 (30): 175-190.

Colque, G.

2017 Tipnis bajo asedio. Fundación Tierra. (14 septiembre 2017), https://tipnisboliviaorg.files.wordpress.com/2018/11/ aca-artc-tierra-00745-2017.pdf (4 octubre 2019).

Correa, S.; Figueroa, C.; Jocelyn Holt, A.; Rolle, C. y Vicuña, M. 2001 Historia del siglo XX chileno. Editorial Sudamericana, Santiago.

De Sousa Santos, B.

2010 Refundación del Estado en América Latina. Perspectivas desde una epistemología del Sur. Lima: Instituto Internacional de Derecho y Sociedad y Programa Democracia y Transformación Global.

Escobar, A.

2014 La invención del desarrollo. Popayán: Universidad de Cauca.

Fundación Solón

2019 Litio Boliviano-¿Industrialización o Extractivismo? Tunapa boletín 108.

Göbel, B. y A. Ulloa (Editoras)

2014 Extractivismo minero en Colombia y América Latina. Bogotá: Universidad Nacional de Colombia.

González-Hidalgo, M.

2018 Disciplinamiento de las subjetividades como estrategia de prevención de incendios: El caso de las plantaciones forestales en el sur de Chile. Perspectivas Rurales Nueva Época 16 (31) 117-141.

Greenberg, G.

2016 Perspectivas locales sobre el litio en el Salar de Uyuni: Niveles de conocimiento y opiniones generales sobre el proyecto que puede determinar el futuro del litio en el mundo. Independent Study Project (ISP) Collection 2301. Disponible en: https://digitalcollections.sit.edu/isp_collection/2301.

Gudynas, E.

2009 Diez tesis urgentes sobre el nuevo extractivismo-contextos y demandas bajo el progresismo sudamericano actual. Editado por CAAP (Centro Andino de Acción Popular) y CLAES (Centro Latino Americano de Ecología Social), pp. 187-225. Quito, Ecuador.

Hernández E., E. y M. Navedo, I. Ana

2011 El Vivir Bien versus hedonismo ético. ¿Fin del dominio a través del consumo? CIDES-UMSA, editado por en: Fara, H. y Vasapollo, L. (Coordinadores). La Paz, Bolivia.

Hope, J.

2016 Losing ground? Extractive-led development versus environmentalism in the Isiboro Secure Indigenous Territory and National Park (TIPNIS), Bolivia. Revista The Extractive Industries and Society 3: 922-929.

Huanacuni Mamani, F.

2010 Buen Vivir/Vivir Bien- Filosofía, políticas, estrategias y experiencias regionales andinas. Coordinadora Andina de Organizaciones Indígenas (CAOI), Primera edición. Lima, Perú.

Lalander, $\mathrm{R}$

2016 Ethnic rights and the dilemma of extractive development in plurinational Bolivia. Revista The International Journal 
of Human Rights: 1-18, doi.org/10.1080/13642987.2016.1 179869.

Lander, E.

2011 El Estado en los actuales procesos de cambio en América Latina: Proyectos complementarios/divergentes en sociedades heterogéneas. Editado por Fundación Rosa Luxemburg/ Albaya Yala. Quito, Ecuador.

Leff, E.

2004 Saber Ambiental. Sustentabilidad, racionalidad, complejidad, poder. Siglo XXI Editores, México.

Lehnert, $\mathrm{M}$.

2013 Llevando el vivir bien en la realidad: La concepción del MAS-IPSP sobre el desarrollo económico en Bolivia. Revista de Ciencias Sociales DECURSOS. Año XI, 27-28: 177-195. Centro de Estudios Superiores Universitarios, Universidad Mayor de San Simón CESU-UMSS. Cochabamba, Bolivia. Lessenich, S.

2019 La sociedad de la externalización. Herder Editorial, $1^{a}$ edición. Barcelona.

López Flores, P.

2014 Neo-extractivismo y Vivir Bien en Bolivia: TIPNIS (Continuidades y conflictos en las políticas de desarrollo en Suramérica. Revista Alternativa 1: 29-44.

López Navia, Á.

2019 Pueblos indígenas en Colombia: Entre el petróleo y la supervivencia étnica. Estudio de caso del pueblo Cofán. Revista Diálogo Andino 60: 127-139.

Lombardi, V.

2018 Litio: hay vida más allá del extractivismo. Nodal (noticias de América Latina y el Caribe). (28 mayo 2018), https://www.nodal.am/2018/05/litio-hay-vida-mas-alla-delextractivismo-por-vanina-lombardi/ (12 septiembre 2019).

Mun, N. K.

2015 La problemática transición boliviana hacia la época postneoliberal: el caso de la economía comunitaria. Revista de Estudios Sociales № 54: 25-38.

Ministerio de Desarrollo Social. Gobierno de Chile

2018 Informe de estimaciones comunales de pobreza, con datos de Casen 2015. Aplicación de metodologías de estimación directa, de estimación para áreas pequeñas (SAE) e imputación de medias por conglomerados (IMC). Serie Documentos Metodológicos Casen $\mathrm{N}^{\circ} 35$. Disponible en: http://observatorio.ministeriodesarrollosocial.gob.cl/documentos/INFORME_estimaciones_pobreza_comunal_2015. pdf (25 julio de 2019).

Nahuelpan, H.

2013 Formación colonial del Estado y desposesión en Ngulumapu. En Ta iñ fijke xipa rakizuameluwün. Historia, colonialismo y resistencia desde el país Mapuche. COMUNIDAD DE HISTORIA MAPUCHE. Temuco: Ediciones Comunidad de Historia Mapuche.
Organización de las Naciones Unidas para la Alimentación y la Agricultura

2016 Casos ejemplares de manejo forestal sostenible en Chile, Costa Rica, Guatemala y Uruguay, http://www.fao. org/3/a-i6003s.pdf (24 junio 2019).

Ostrom, E.

2009 A general framework for analyzing sustainability of socio-ecological systems. Science 325: 419-422.

Pacasi Mamani, R.; Argandoña, Molina W.; Ortiz Chirino, F. y Saaresranta, T.

2012 Vivir Bien -significados y representaciones desde la vida cotidiana- Cuatro miradas: Jesús de Machaca (La Paz), San Ignacio de Mojos (Beni), La Guardia (Santa Cruz) y, zona sur Cochabamba. Fundación para la Investigación Estratégica en Bolivia (PIEB). La Paz, Bolivia.

Paz, S.

2012 La marcha indígena del Tipnis en Bolivia y su relación con los modelos extractivos de América del Sur (junio 2012), doi: 10.22409/GEOgraphia2011.1326.a13623 (7 noviembre 2019).

Pinol, A. (Ed.)

2015 Democracia versus Neoliberalismo. 25 años de neoliberalismo en Chile. Santiago: LOM.

Programa de Gobierno MAS-IPSP, 2010-2015

Sin fecha. Bolivia-País Líder. Documento oficial del Estado Plurinacional de Bolivia.

Puente, R.

2011 Vivir Bien y descolonización. Editado por Fara, H. y Vasapollo, L. (Coordinadores). CIDES-UMSA. La Paz, Bolivia.

Radio Duna

2017 Iceberg del porte de 10 ciudades se desprende de la Antártida. (12 julio 2017), http://www.duna.cl/ noticias/2017/07/12/iceberg-del-porte-de-10-ciudades-sedesprende-de-la-antartica/ (13 april 2019).

Rivera Cusicanqui, S.

2018 Un mundo ch'ixi es posible. Ensayos desde un presente en crisis. Buenos Aires: Tinta Limón.

Simbaña, F.

2011 El sumak kawsay como proyecto político. Editado por Fundación Rosa Luxemburg/ Albaya Yala. Quito, Ecuador.

Ströbele-Gregor, J.

2013 El proyecto estatal del litio en Bolivia-Expectativas, desafíos y dilemas. Revista Nueva Sociedad, 244: 74-83.

Svampa, M.

2016 Debates Latinoamericanos: Indianismo, Desarrollo, Dependencia y Populismo. Buenos Aires: Edhasa.

Villalba-Eguiluz, Unai C. y Etxano, I.

2017 Buen Vivir vs. Development (II): The Limits of (Neo)Extractivism. Revista Ecological Economics 138: 1-11. 


\section{Notas}

1 Paradojalmente, en julio de 2017 se informa que en la Antártica se derrite "uno de los mayores iceberg registrados", lo que podemos considerar como una cruel metáfora de la política y la economía, que han impedido el establecimiento de acuerdos, normas y pautas de acción para el resguardo local y global de los recursos naturales. "El bloque de hielo, de 5.800 kilómetros cuadrados y un billón de toneladas, será probablemente bautizado como A68, según los científicos del proyecto Midas, una misión británica que investiga los efectos del calentamiento global en el continente helado" (Radio Duna, 2017).

2 Movimiento al Socialismo-Instrumento Político por la Soberanía de los Pueblos. Partido político que gobernó, bajo el liderazgo de Evo Morales como presidente, a partir de finales de 2005 al 10 de noviembre de 2019 en Bolivia.

3 Artículo 8 de la Constitución Política del Estado de 2009: I. El Estado asume y promueve como principios éticos-morales de la sociedad plural: ama qhilla, ama llulla, ama suwa (no seas flojo, no seas mentiroso ni seas ladrón), suma qamaña (vivir bien), ñandereko (vida armoniosa), teko kavi (vida buena), ivi maraei (tierra sin mal) y qhapaj ñan (camino o vida noble). II. El Estado se sustenta en los valores de unidad, igualdad, inclusión, dignidad, libertad, solidaridad, reciprocidad, respeto, complementariedad, armonía, transparencia, equilibrio, igualdad de oportunidades, equidad social y de género en la participación, bienestar común, responsabilidad, justicia social, distribución y redistribución de los productores y bienes sociales, para vivir bien.

4 Las cifras vigentes indican que las comunas con mayor superficie dedicada a las plantaciones forestales presentan las siguientes cifras de pobreza multidimensional: Curanilahue 23,2\%; Collipulli 35,9\%; Ránquil 30\% (MDS, 2018).

5 Consultoría V26: "Análisis de la problemática social asociada al sector de plantaciones y a la industria de celulosa y papel en el sur de Chile", WWF Chile.

6 Ver como ejemplo las plataformas http://www.mapuexpress.org y https://resumen.cl.

7 Territorio Indígena y Parque Nacional Isiboro Sécure (Tipnis) (Colque en Fundación Tierra, 2017).

8 Sistema homologado en Chile por medio del Estándar CERTFOR.

$9 \quad$ Confederación de Pueblos Indígenas de Bolivia.

10 Consejo Nacional de Ayllus y Markas del Qullasuyu. 